\title{
BMJ Open Prehospital antiplatelet use and functional status on admission of patients with non-haemorrhagic moyamoya disease: a nationwide retrospective cohort study (J-ASPECT study)
}

To cite: Onozuka $D$, Hagihara A, Nishimura K, et al. Prehospital antiplatelet use and functional status on admission of patients with non-haemorrhagic moyamoya disease: a nationwide retrospective cohort study (J-ASPECT study). BMJ Open 2016;6:e009942. doi:10.1136/bmjopen-2015009942

- Prepublication history and additional material is available. To view please visit the journal (http://dx.doi.org/ 10.1136/bmjopen-2015009942).

Received 9 September 2015 Revised 27 November 2015 Accepted 18 January 2016

CrossMark

For numbered affiliations see end of article.

\section{Correspondence to}

Professor Koji lihara; kiihara@ns.med.kyushu-u. ac.jp

\section{ABSTRACT}

Objectives: To elucidate the association between antiplatelet use in patients with non-haemorrhagic moyamoya disease before hospital admission and good functional status on admission in Japan.

Design: Retrospective, multicentre, non-randomised, observational study.

Setting: Nationwide registry data in Japan.

Participants: A total of 1925 patients with nonhaemorrhagic moyamoya disease admitted between 1 April 2012 and 31 March 2014 in Japan.

Main outcome measure: We performed propensity score-matched analysis to examine the association between prehospital antiplatelet use and no significant disability on hospital admission, as defined by a modified Rankin Scale score of 0 or 1.

Results: Propensity-matched patients who received prehospital antiplatelet drugs were associated with a good outcome on hospital admission (OR adjusted for all covariates, $3.82 ; 95 \% \mathrm{Cl} 1.22$ to 11.99 ) compared with those who did not receive antiplatelet drugs prior to hospital admission.

Conclusions: Prehospital antiplatelet use was significantly associated with good functional status on hospital admission among patients with nonhaemorrhagic moyamoya disease in Japan. Our results suggest that prehospital antiplatelet use should be considered when evaluating outcomes of patients with non-haemorrhagic moyamoya disease.

\section{INTRODUCTION}

Moyamoya disease is an uncommon condition characterised by progressive stenosis of

\section{Strengths and limitations of this study}

- This is the first report of a relationship between prehospital antiplatelet use and a good outcome of non-haemorrhagic moyamoya disease.

- Our findings were derived from nationwide registry data from 327 certified training medical institutions in Japan, and the present study provides a representative patient population in Japan.

- Prehospital antiplatelet use was not random.

- Moyamoya disease is uncommon, and the sample size for propensity-matched analysis was relatively small.

the terminal portion of the internal carotid artery and its main branches. ${ }^{1}{ }^{2}$ Its aetiology is unknown; it is most common in countries in East Asia such as Japan and Korea, and this disease has been increasingly recognised in the USA and other Western countries. ${ }^{3-5}$ Moyamoya disease is the most common paediatric cerebrovascular disease in Japan, with an estimated annual prevalence of approximately 3 cases per 100000 people. ${ }^{367}$ The reported incidence of moyamoya disease in Europe is $1 / 10$ th that in Japan. ${ }^{8}{ }^{9}$ In the USA, the incidence is 0.086 cases per 100000 persons, and ethnicityspecific incidence rate ratios compared with Caucasians were 4.6 for Asian Americans, 2.2 for African Americans and 0.5 for Hispanics. ${ }^{10}$ There have been no doubleblind randomised controlled trials regarding 
treatment, and there is uncertainty about the use of antiplatelet agents in the management of moyamoya disease. ${ }^{11}$

The oral administration of antiplatelet agents is believed to reduce the incidence and improve prognosis of patients with non-haemorrhagic moyamoya disease. The use of antithrombotic drugs such as aspirin, ozagrel, argatroban and heparin has been recommended in adult patients with moyamoya disease manifesting as cerebral infarction. ${ }^{3}{ }^{12}$ However, there is insufficient evidence for using these drugs, especially for asymptomatic patients with moyamoya. ${ }^{3} 11$ In paediatric patients, antiplatelet therapy with aspirin alone has been reported to be effective; however, it may also increase the risk of Reye syndrome. ${ }^{3}{ }^{14}$ Findings from previous studies were not consistent, and the effectiveness of antiplatelet drugs for moyamoya disease has not been established. There have been no previous studies regarding the effects of prehospital antiplatelet use on functional status on admission in patients with nonhaemorrhagic moyamoya disease.

We investigated the association between the use of antiplatelet drugs and good functional status in patients with non-haemorrhagic moyamoya disease admitted to hospitals, in a multicentre database from the Acute Stroke care capacity for Proper dEsignation of Comprehensive stroke cenTer in Japan (J-ASPECT study).

\section{METHODS}

\section{Data collection}

This study was based on multicentre nationwide data from a 2-year survey (J-ASPECT study) of patients with moyamoya disease in Japan. A consecutive and retrospective database of all patients with stroke admitted to the hospital was started in 2012. Among 847 certified training institutions of the Japan Neurosurgical Society, Japanese Society of Neurology and/or Japan Stroke Society throughout the country, 327 institutions participated in this study. The J-ASPECT database includes the clinical data and disease classification of all patients with stroke obtained from the participating institutions. Disease classification is recorded according to the International Classification of Diseases, 10th revision (ICD-10). Detailed information of the J-ASPECT database has been provided elsewhere. ${ }^{15-17}$ Data of all patients with moyamoya disease admitted to the hospital between 1 April 2012 and 31 March 2014 with a diagnosis of moyamoya disease according to the ICD-10 code I67.5 were used.

\section{Study design and patients}

This was a retrospective observational study. The requirement for written informed consent was waived. Patient records and other patient information remained anonymous and were de-identified prior to analysis. All methods were carried out in accordance with approved guidelines and regulations.

\section{Identification of moyamoya disease}

Moyamoya disease was diagnosed by patients' medical history and radiological examinations according to the criteria of the guidelines for diagnosis and treatment of moyamoya disease (ie, spontaneous occlusion of the circle of Willis). ${ }^{3}{ }^{18-22}$ Diagnostic criteria of moyamoya disease were characterised by (1) stenosis or occlusion at the terminal portions of the internal carotid artery, or the proximal areas of the anterior or middle cerebral arteries, (2) presence of abnormal vascular networks in the arterial territories near the occlusive or stenotic lesions and (3) bilateral lesion. A definite diagnosis was based on CT examination or MRI/angiography findings without conventional catheter angiography. Patients were evaluated to exclude all other similar cerebrovascular lesions associated with atherosclerosis, autoimmune disease, meningitis, brain tumours, Down syndrome, neurofibromatosis type-1, traumatic brain injury, cranial irradiation, etc.

\section{Study variables}

Study variables are listed by prehospital antiplatelet use status in table 1 . Collected data on patients with moyamoya disease included demographic data (age and sex); body mass index (BMI); smoking history; and a medical history of hypertension, diabetes mellitus and hyperlipidaemia. Prehospital antiplatelet use (aspirin, clopidogrel and cilostazol) was identified from outpatient procedure information of the participating hospitals based on the Diagnosis Procedure Combination (DPC) Database. The day of the week was also included as a categorical explanatory variable because previous studies reported higher mortality for patients with stroke admitted on weekends. ${ }^{23}{ }^{24}$ The number of days from stroke onset was included as a categorical variable $(0-3,4-7$, $\geq 8$ days and asymptomatic or not available). Assessment of coma and impaired consciousness on hospital admission was measured using the Japan Coma Scale. ${ }^{17}{ }^{25}$ The modified Rankin Scale score was used on admission as a measure of disability and functional status after stroke, with values ranging from 0 (no symptoms) to 5 (severe disability and bedridden) and 6 (death) ${ }^{26}$ The modified Rankin Scale was evaluated by local neurologists who were blinded to treatment assignment. Comorbidity was assessed with the Charlson Comorbidity Index score. ${ }^{27}$ Data on Comprehensive Stroke Centre (CSC) capability were used as a measure of hospital ability. CSC capability was defined by the CSC score, which was recommended by members of the Brain Attack Coalition, ${ }^{28}$ for establishing CSCs capable of delivering a wide variety of specialised care to seriously ill patients with stroke and cerebrovascular disease. Details have been published previously. ${ }^{15-17}$

\section{End points}

The primary end point was the proportion of patients who had a good functional status defined by a modified Rankin Scale score of 0-1 (ie, symptom free or residual 
Table 1 Baseline characteristics of patients with moyamoya disease according to prehospital antiplatelet use $(n=1925)$

\begin{tabular}{|c|c|c|c|}
\hline Characteristics & $\begin{array}{l}\text { No } \\
\text { antiplatelet } \\
\text { use } \\
(n=1223)\end{array}$ & $\begin{array}{l}\text { Antiplatelet } \\
\text { use }(n=702)\end{array}$ & p Value \\
\hline Male, number (\%) & $457(37.4)$ & $218(31.1)$ & 0.005 \\
\hline $\begin{array}{l}\text { Age, mean }(S D), \\
\text { years }\end{array}$ & $30.8(19.3)$ & $34.5(19.0)$ & $<0.001$ \\
\hline $\begin{array}{l}\text { Body mass index, } \\
\text { mean (SD) }\end{array}$ & $21.8(5.6)$ & $22.5(4.8)$ & 0.005 \\
\hline $\begin{array}{l}\text { Smoking history, } \\
\text { number (\%) }\end{array}$ & $258(21.1)$ & $200(28.5)$ & $<0.001$ \\
\hline \multicolumn{4}{|c|}{ Medical history, number (\%) } \\
\hline Hypertension & $177(14.5)$ & $166(23.7)$ & $<0.001$ \\
\hline $\begin{array}{l}\text { Diabetes } \\
\text { mellitus }\end{array}$ & $73(6.0)$ & $38(5.4)$ & 0.615 \\
\hline Hyperlipidaemia & $97(7.9)$ & $104(14.8)$ & $<0.001$ \\
\hline $\begin{array}{l}\text { Emergency } \\
\text { hospitalisation, } \\
\text { number (\%) }\end{array}$ & $100(8.2)$ & $80(11.4)$ & 0.020 \\
\hline \multicolumn{4}{|c|}{ Day of the week of hospital admission, number (\%) } \\
\hline Sunday & $42(3.4)$ & $24(3.4)$ & 0.078 \\
\hline Monday & $312(25.5)$ & $203(28.9)$ & \\
\hline Tuesday & $272(22.2)$ & $128(18.2)$ & \\
\hline Wednesday & $239(19.5)$ & $139(19.8)$ & \\
\hline Thursday & 175 (14.3) & $111(15.8)$ & \\
\hline Friday & $166(13.6)$ & 79 (11.3) & \\
\hline Saturday & $17(1.4)$ & $18(2.6)$ & \\
\hline \multicolumn{4}{|c|}{ Number of days from stroke onset, number (\%) } \\
\hline $0-3$ & $90(7.4)$ & $62(8.8)$ & 0.677 \\
\hline $4-7$ & $11(0.9)$ & $5(0.7)$ & \\
\hline$\geq 8$ & $632(51.7)$ & $355(50.6)$ & \\
\hline $\begin{array}{l}\text { Asymptomatic or } \\
\text { not available }\end{array}$ & $490(40.1)$ & $280(39.9)$ & \\
\hline \multicolumn{4}{|c|}{ Japan Coma Scale on hospital admission, number (\%) } \\
\hline 0 & 1169 (95.6) & 665 (94.7) & 0.105 \\
\hline $1-3$ & 47 (3.8) & $37(5.3)$ & \\
\hline $10-30$ & $3(0.3)$ & $0(0.0)$ & \\
\hline $100-300$ & $4(0.3)$ & $0(0.0)$ & \\
\hline
\end{tabular}

Charlson Comorbidity Index score on hospital admission, number (\%)

$\begin{array}{lccc}1 & 718(58.7) & 351(50.0) & <0.001 \\ 2 & 236(19.3) & 142(20.2) & \\ 3 & 128(10.5) & 108(15.4) & \\ 4 & 99(8.1) & 62(8.8) & \\ 5 & 25(2.0) & 33(4.7) & \\ 6 & 15(1.2) & 5(0.7) & \\ 7 & 2(0.2) & 1(0.1) & \\ \text { CSC score, mean } & 18.1(2.7) & 18.2(3.5) & 0.585 \\ \text { (SD) } & & \\ \text { Modified Rankin Scale score on hospital admission, } \\ \text { number (\%) } \\ 0 & 652(53.3) & 369(52.6) & 0.028 \\ 1 & 409(33.4) & 266(37.9) & \\ 2 & 102(8.3) & 50(7.1) & \\ 3 & 39(3.2) & 10(1.4) & \\ 4 & 15(1.2) & 7(1.0) & \\ 5 & 6(0.5) & 0(0.0) & \end{array}$

CSC, Comprehensive Stroke Centre. symptoms with no loss of activity) ${ }^{29}$ on hospital admission. All modified Rankin Scale assessments were performed by study investigators involved in treating patients who were unaware of the treatment assignment.

\section{Statistical analysis}

Data that fulfilled the inclusion criteria of the modified Rankin Scale score were analysed. Data for all cases were fitted to three types of unconditional logistic regression models using the modified Rankin Scale score on hospital admission as a dependent variable.

As antiplatelet use before hospital admission was not random in the patient population, we developed a propensity score for antiplatelet use before hospital admission, and controlled for potential confounding factors and selection bias. ${ }^{30} 31$ The propensity score for prehospital antiplatelet use was determined by multivariate logistic regression analysis without considering patient outcomes. Specifically, a full non-parsimonious logistic regression model was fitted with prehospital antiplatelet use as a dependent variable, which included all variables as independent variables; shown in table 1 . The propensity score for antiplatelet use before hospital admission was calculated from the logistic regression equation for each patient. This propensity score represented the probability that a patient with moyamoya disease would be given an antiplatelet agent before hospital admission. Based on the propensity score, patients with moyamoya disease who received antiplatelet agents before hospital admission were matched with unique control patients who were not given such agents before hospital admission. Using data for the propensity-matched patients, four types of conditional logistic regression models were fitted with a modified Rankin Scale score on hospital admission as a dependent variable.

The two-sided significance level for all tests was $\mathrm{p}<0.05$. All statistical analyses were performed using Stata V.13.1 (Stata Corp, College Station, Texas, USA) and R, V.3.1.2 ( $\mathrm{R}$ Core Team, $\mathrm{R}$ Foundation for Statistical Computing, Vienna, Austria).

\section{RESULTS}

\section{Patients' characteristics}

We analysed 1925 patients with non-haemorrhagic moyamoya disease (figure 1 and table 1 ). The mean patient age was $28.9 \pm 19.9$ years in men and $33.9 \pm 18.7$ years in women, and the distribution of age at onset by sex has been suggested to have two peaks (see online supplementary figure S1). Significant differences were found between the antiplatelet use and no antiplatelet use groups with respect to sex, age, BMI, smoking history, medical history of hypertension and hyperlipidaemia, emergency hospitalisation, Charlson Comorbidity Index score on hospital admission and modified Rankin Scale score on hospital admission. Patients with moyamoya treated with prehospital antiplatelet therapy were significantly older, had a higher BMI, and had a higher 


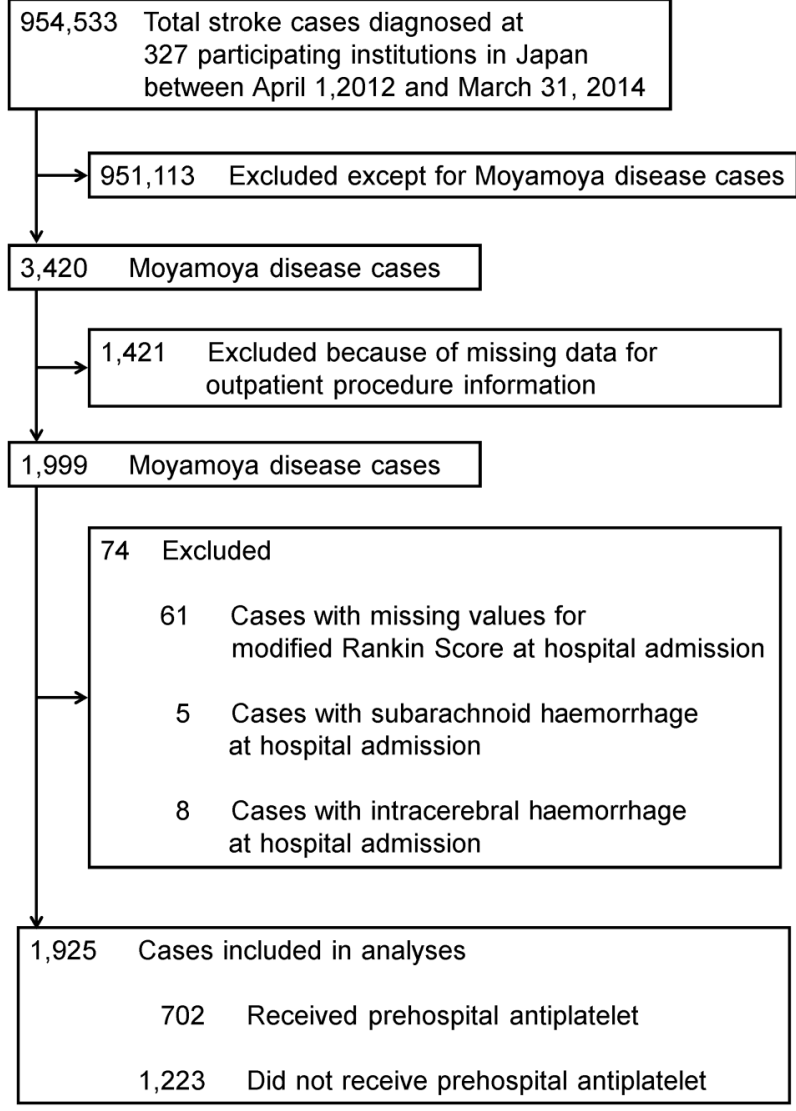

Figure 1 Study participant selection.

incidence of hypertension, hyperlipidaemia and a smoking history.

\section{Antiplatelet use and outcomes}

Relationships between prehospital antiplatelet use and outcomes among all patients with non-haemorrhagic moyamoya disease are shown in table 2. In the unadjusted model, there was a significant association between prehospital antiplatelet use and good functional status on hospital admission (OR, 1.45; 95\% CI 1.07 to 1.96). After adjusting for all covariates, there was a similar association between prehospital antiplatelet use

Table 2 Unconditional logistic regression analysis of prehospital antiplatelet use and outcomes among patients with moyamoya disease $(n=1925)$

\begin{tabular}{lll}
\hline Analysis & OR & (95\% Cl) \\
\hline Unadjusted & 1.45 & (1.07 to 1.96) \\
Adjusted for selected variables * & 1.53 & $(1.12$ to 2.10$)$ \\
Adjusted for all covariatest & 1.52 & (1.11 to 2.09)
\end{tabular}

*Selected variables included sex, age, body mass index, smoking history, medical history of hypertension, medical history of diabetes mellitus, medical history of hyperlipidaemia, emergency hospitalisation, day of the week of hospital admission, number of days from stroke onset, Japan Coma Scale on hospital admission, and Charlson Comorbidity Index score on hospital admission. †All covariates included selected variables plus Comprehensive Stroke Centre (CSC) score. and good outcome on hospital admission (OR, 1.52; $95 \%$ CI 1.11 to 2.09 ).

\section{Antiplatelet use and outcomes in propensity-matched patients}

To calculate the propensity score, a full nonparsimonious logistic regression model was fit. This model yielded a $\mathrm{C}$ statistic of 0.724 , which indicated a strong ability to differentiate between those who did and those who did not use antiplatelet agents before hospital admission. The propensity score ranged from 0.216 to 0.632 , which indicated that the probability of antiplatelet use before hospital admission by a patient with nonhaemorrhagic moyamoya disease would be between 0.216 and 0.632 . In this study, 289 patients who received antiplatelet agents were matched with 289 patients who did not receive such treatment (table 3 ). There were no significant differences in any of the variables between patients who received antiplatelet agents and those who did not, indicating that these propensity-matched patients were well matched. Our propensity score matching reduced standardised differences for all covariates below $10 \%$ in absolute value, demonstrating substantial improvement in covariate balance across the treatment groups (see online supplementary figure S2) ${ }^{32} 33$

Relationships between prehospital antiplatelet use and outcomes among propensity-matched patients with nonhaemorrhagic moyamoya disease are shown in table 4 . In the unadjusted model, there was a significant relationship between prehospital antiplatelet use and good functional status on hospital admission (OR, 1.71; 95\% CI 1.00 to 2.94). After adjusting for all covariates, prehospital antiplatelet use was significantly associated with good outcomes on hospital admission (OR, 3.82; 95\% CI 1.22 to 11.99). The positive association became increasingly more evident after adjusting for selected variables, and after adjusting for all variables.

\section{DISCUSSION}

Most importantly, our results suggested that, based on propensity-matched analysis controlling for selection bias and confounding factors, there was evidence for good outcomes on hospital admission among patients with non-haemorrhagic moyamoya disease with prehospital antiplatelet use. Moreover, these positive associations became increasingly evident after adjusting for selected variables, and after adjusting for all variables. This is the first report of a relationship between prehospital antiplatelet use and a good outcome of non-haemorrhagic moyamoya disease. Our results suggested that prehospital antiplatelet use should be considered when evaluating outcomes in cases of non-haemorrhagic moyamoya disease, and this might be of value in both the clinical management of non-haemorrhagic moyamoya disease and in the ability of patients to cope with the disease.

Appropriate medical treatments for moyamoya disease are not currently available. ${ }^{11}{ }^{34}$ Therefore, it is widely 
Table 3 Baseline characteristics of patients with moyamoya disease according to prehospital antiplatelet use in propensity-matched patients $(n=578)$

\begin{tabular}{|c|c|c|c|}
\hline Characteristics & $\begin{array}{l}\text { No } \\
\text { antiplatelet } \\
\text { use } \\
(n=289)\end{array}$ & $\begin{array}{l}\text { Antiplatelet } \\
\text { use }(n=289)\end{array}$ & p Value \\
\hline Male, number (\%) & $103(35.6)$ & $93(32.2)$ & 0.380 \\
\hline $\begin{array}{l}\text { Age, mean (SD), } \\
\text { years }\end{array}$ & 31.3 (18.9) & 30.9 (18.8) & 0.796 \\
\hline $\begin{array}{l}\text { Body mass index, } \\
\text { mean (SD) }\end{array}$ & $22.1(5.7)$ & $22.0(4.5)$ & 0.780 \\
\hline $\begin{array}{l}\text { Smoking history, } \\
\text { number }(\%)\end{array}$ & $66(22.8)$ & $52(18.0)$ & 0.149 \\
\hline \multicolumn{4}{|c|}{ Medical history, number (\%) } \\
\hline Hypertension & $36(12.5)$ & $49(17.0)$ & 0.127 \\
\hline $\begin{array}{l}\text { Diabetes } \\
\text { mellitus }\end{array}$ & $13(4.5)$ & $17(5.9)$ & 0.453 \\
\hline Hyperlipidaemia & $28(9.7)$ & $26(9.0)$ & 0.775 \\
\hline $\begin{array}{l}\text { Emergency } \\
\text { hospitalisation, } \\
\text { number (\%) }\end{array}$ & $27(9.3)$ & $25(8.7)$ & 0.771 \\
\hline \multicolumn{4}{|c|}{ Day of the week of hospital admission, number (\%) } \\
\hline Sunday & $13(4.5)$ & $8(2.8)$ & 0.336 \\
\hline Monday & $75(26.0)$ & $73(25.3)$ & \\
\hline Tuesday & $59(20.4)$ & $62(21.5)$ & \\
\hline Wednesday & $62(21.5)$ & $59(20.4)$ & \\
\hline Thursday & $30(10.4)$ & $47(16.3)$ & \\
\hline Friday & $42(14.5)$ & $31(10.7)$ & \\
\hline Saturday & $8(2.8)$ & $9(3.1)$ & \\
\hline \multicolumn{4}{|c|}{ Number of days from stroke onset, number (\%) } \\
\hline $0-3$ & $21(7.3)$ & $24(8.3)$ & 0.209 \\
\hline $4-7$ & $4(1.4)$ & $0(0.0)$ & \\
\hline$\geq 8$ & $159(55.0)$ & $153(52.9)$ & \\
\hline $\begin{array}{l}\text { Asymptomatic or } \\
\text { not available }\end{array}$ & $105(36.3)$ & $112(38.8)$ & \\
\hline \multicolumn{4}{|c|}{ Japan Coma Scale on hospital admission, number (\%) } \\
\hline 0 & $278(96.2)$ & $273(94.5)$ & 0.261 \\
\hline $1-3$ & $9(3.1)$ & $16(5.5)$ & \\
\hline $10-30$ & $1(0.4)$ & $0(0.0)$ & \\
\hline $100-300$ & $1(0.4)$ & $0(0.0)$ & \\
\hline
\end{tabular}

Charlson Comorbidity Index score on hospital admission, number (\%)

\begin{tabular}{lrrr}
1 & $167(57.8)$ & $168(58.1)$ & 0.522 \\
2 & $63(21.8)$ & $54(18.7)$ & \\
3 & $27(9.3)$ & $34(11.8)$ & \\
4 & $21(7.3)$ & $18(6.2)$ & \\
5 & $7(2.4)$ & $13(4.5)$ & \\
6 & $4(1.4)$ & $2(0.7)$ & \\
7 & $0(0.0)$ & $0(0.0)$ & \\
CSC score, mean & $18.0(2.8)$ & $18.1(3.5)$ & 0.944 \\
(SD) & & \\
Modified Rankin Scale score on hospital admission, \\
number (\%) \\
5 \\
0 & $142(49.1)$ & $148(51.2)$ & 0.261 \\
1 & $105(36.3)$ & $114(39.5)$ & \\
2 & $32(11.1)$ & $19(6.6)$ & \\
3 & $4(1.4)$ & $6(2.1)$ & \\
4 & $5(1.7)$ & $2(0.7)$ \\
5 & $1(0.4)$ & $0(0.0)$ & \\
\hline CSC, Comprehensive Stroke Centre. &
\end{tabular}

CSC, Comprehensive Stroke Centre.
Table 4 Conditional logistic regression analysis of prehospital antiplatelet use and outcomes among propensity-matched patients with moyamoya disease $(n=578)$

\begin{tabular}{|c|c|c|}
\hline Analysis & OR & $(95 \% \mathrm{Cl})$ \\
\hline & & \\
\hline ity & & \\
\hline $\begin{array}{l}\text { ed for propensity and } \\
\text { d variables* }\end{array}$ & & (1.28 to 12.96$)$ \\
\hline $\begin{array}{l}\text { Adjusted for propensity and all } \\
\text { covariates } \dagger\end{array}$ & 3.82 & $(1.22$ \\
\hline \multicolumn{3}{|c|}{$\begin{array}{l}\text { *Selected variables included sex, age, body mass index, smoking } \\
\text { history, medical history of hypertension, medical history of } \\
\text { diabetes mellitus, medical history of hyperlipidaemia, emergency } \\
\text { hospitalisation, day of the week of hospital admission, number of } \\
\text { days from stroke onset, Japan Coma Scale on hospital admission, } \\
\text { and Charlson Comorbidity Index score on hospital admission. } \\
\text { †All covariates included selected variables plus Comprehensive } \\
\text { Stroke Centre (CSC) score. }\end{array}$} \\
\hline
\end{tabular}

believed that direct and indirect cerebrovascular revascularisation procedures are advisable, and they remain the most beneficial form of therapy despite the lack of systematic evaluation protocols for operative treatment. ${ }^{3}$ We found that prehospital antiplatelet use was a significant predictor of good outcome on hospital admission for non-haemorrhagic moyamoya disease. Our findings also suggested the importance of early identification of high-risk individuals predisposed to non-haemorrhagic moyamoya disease and early medical treatment to reduce the risk of ischaemic stroke and transient ischaemic attack.

Antiplatelet use before hospital admission was consistently a significant and positive predictor of outcome on hospital admission in the four statistical models. Aspirin is widely recommended as antiplatelet therapy for patients with non-haemorrhagic moyamoya disease. ${ }^{3}$ Additionally, a recent systematic review established that aspirin at a dose of 160-300 mg daily, started within $48 \mathrm{~h}$ of the onset of stroke symptoms, reduced the risk of early recurrent ischaemic stroke without a major risk of early haemorrhagic complications, and long-term outcomes were also improved. ${ }^{35}$ Recent studies also showed that microembolic signals are associated with recent ischaemic symptoms and progression of arterial lesion, and independently predict cerebral ischaemic events among patients with moyamoya disease. ${ }^{36} 37$ European cohort studies also indicated that embolic infarctions are sometimes observed in the beginning of moyamoya disease, and haemodynamic compromise was also shown in patients with high-intensity transient signals. ${ }^{38} 39$ Thus, aspirin may help in embolic strokes, not in haemodynamic ischaemia. Clopidogrel has also been considered as an alternative in cases where aspirin is not tolerated or fails, ${ }^{40}$ and a recent systematic review indicated that an alternative antiplatelet agent should be considered in people who cannot tolerate aspirin, although evidence for other agents is inadequate. ${ }^{35}$ Also, a large-scale trial indicated that antiplatelets are 
beneficial for all forms of intracranial stenosis. ${ }^{41-44}$ Our results were consistent with previous findings. However, other trials of combination therapy with clopidogrel and aspirin did not show a reduction in the risk of ischaemic events but did show an increased risk of haemorrhage. ${ }^{45-47}$ This discrepancy may have been due to differences in the target population; mainly patients with transient ischaemic attack and minor stroke were included in the previous studies.

This study had several other notable findings. First, in the propensity-matched patients, a significant positive association was also detected after adjusting for all covariates (table 4). Our findings are derived from four different models, and all analyses consistently showed that prehospital antiplatelet use was independently associated with good outcomes on hospital admission among patients with non-haemorrhagic moyamoya disease. Our results showed no clear heterogeneity of a treatment effect with all covariates. This suggests that patients with moyamoya disease are likely to benefit from prehospital antiplatelet therapy. Second, overall, $88.1 \%$ of patients in the antiplatelet group had a good functional outcome on hospital admission. A similar basic pattern was found among propensity-matched patients. The natural course of moyamoya disease is not fully understood because there have been few studies on the progression of the disease. ${ }^{1}$ Thus, our results suggest the importance of prehospital antiplatelet use for the prognosis of moyamoya disease. However, previous studies have suggested that the frequency of cerebrovascular events, including ischaemic and haemorrhagic stroke, is high in adults with moyamoya disease who are treated conservatively. ${ }^{9} 4849$ This discrepancy may be due to the effects of varying factors for CSC capability and mutual confounding for antiplatelet use; these were controlled for in the present study, but not in previous studies. Studies of a more appropriate design and further discussion of antiplatelet-specific issues are needed, and very large randomised trials are required.

Understanding the effects of antiplatelet use for moyamoya disease is important for planning the treatment of this disease. Our results suggested a potential benefit of prehospital antiplatelet use if the diagnosis can be made earlier. Aspirin, especially, is inexpensive, easy to administer and safe, which increases its potential public health use globally. A previous worldwide survey showed that the majority of non-Asian experts recommended longterm antiplatelet therapy for moyamoya disease, although, among Asian experts, this is an unusual approach to treating this disease. ${ }^{11}$ If antiplatelet treatment prevents a poor outcome, then more patients could be saved worldwide with substantial numbers avoiding long-term disability. Especially, the use of aspirin among Asian experts would benefit, as the incidence of moyamoya disease is higher among Japanese and Korean populations, while intracranial atherosclerosis (ICAS) is more common in Chinese and other Asian groups. ${ }^{50-52}$
Our study had several methodological limitations. First, prehospital antiplatelet use was not random. Considering possible non-random use of prehospital antiplatelet drugs, we performed a propensity analysis and rigorously adjusted the data for selection bias and confounding factors. Nevertheless, this approach can only partially control and adjust for factors actually measured, and cannot account for non-observed variables. Thus, any hidden bias due to latent variables may remain after matching, whereas randomised allocation can control for both, known and unknown confounding factors, and avoid the introduction of bias.

Second, the reason why patients with moyamoya with prehospital antiplatelet use had a higher percentage of risk factors of cerebrovascular diseases such as hypertension, hyperlipidaemia and a smoking history remains unknown. Moyamoya disease is often associated with hypertension, which has been attributed to stenosis of the renal arteries. Recently, a polymorphism of RNF213, the susceptibility gene for moyamoya disease, was reported to be associated with blood pressure. ${ }^{53}$ However, clinical progression in adult patients with asymptomatic moyamoya disease was associated with decreased vascular reserve, smoking and hypertension. ${ }^{54}$ In this study, patients with moyamoya with haemorrhagic presentation were excluded based on comorbid conditions on admission. Therefore, it is reasonable to assume that the index stroke in these cases was mild ischaemic stroke or transient ischaemic attack, and patients with prehospital antiplatelet use may be those who were considered high risk for clinical or disease progression, or recurrence of ischaemic stroke or attacks in the participating hospitals.

Third, moyamoya disease is uncommon, and the sample size for propensity-matched analysis was relatively small. In Japan, the Japan Adult Moyamoya (JAM) Trial was the largest, multicentre, retrospective, randomised, controlled trial that has been conducted since 2001. ${ }^{26}$ However, this study has been conducted by 22 medical institutions, and it only focused on adult haemorrhagic patients with moyamoya. In addition, a recent study of the JAM trial showed that 213 patients were assessed for eligibility from January 2001 to June $2008 .^{26}$ In contrast, our findings were derived from nationwide registry data from 327 certified training medical institutions in Japan, and the study model used 1925 patients. Thus, we believe that the present study provides a more representative patient population in Japan.

Fourth, owing to the limited number of antiplatelet dose measurements available did not allow individual categorisation by prehospital antiplatelet use, appropriate dose-response estimates were not possible. Additional analyses using dose may provide further insight into the outcome associated with prehospital antiplatelet use.

Fifth, the period we analysed was short (ie, 2 years, from 1 April 2012 to 31 March 2014). The epidemiological features in moyamoya disease have changed over time; for example, for reasons not yet understood, the 
incidence of paediatric moyamoya disease has begun to decrease. Additionally, we could not consider the time period in the progressive nature of moyamoya disease for each patient. These factors indicate a need for more precise modelling of the effects of antiplatelet use on moyamoya disease, and will be a critical focus of future studies.

Sixth, there are controversial issues regarding the treatment of moyamoya disease, especially for asymptomatic adult patients with moyamoya disease. Asymptomatic moyamoya disease in adults is not a permanent stable disease, and the definition and clinical features are still unclear. This study included asymptomatic cases, and cases without a specific interval between the index attack and admission, which accounted for up to $40 \%$ of cases; thus, further study is required to clarify detailed information on their mode of presentation, which may affect their functional status, such as headache, remote mild ischaemic attack, or non-specific symptoms related to haemodynamic insufficiency caused by inadequate collaterals in patients with moyamoya disease. Additionally, this study might include patients who received solely medical management prior to surgical treatment, and data on in-hospital treatment were not included in the analysis. Thus, we could not control for the effects of in-hospital medical procedures, such as surgical treatment. Our results should be interpreted with caution in this respect.

In conclusion, this study provided the first evidence that prehospital antiplatelet use is significantly associated with a good outcome among patients with non-haemorrhagic moyamoya disease in Japan. Prehospital antiplatelet use should be considered when evaluating the outcome of patients with non-haemorrhagic moyamoya disease.

\section{Author affiliations}

${ }^{1}$ Department of Health Communication, Graduate School of Medical Sciences, Kyushu University, Fukuoka, Japan

${ }^{2}$ Department of Preventive Medicine and Epidemiologic Informatics, National Cerebral and Cardiovascular Center, Osaka, Japan

${ }^{3}$ Clinical Research Center, Nagoya Medical Center, Aichi, Japan

${ }^{4}$ Department of Integrative Stroke Imaging Center, National Cerebral and

Cardiovascular Center, Osaka, Japan

${ }^{5}$ Department of Neurosurgery, Iwate Medical University, Iwate, Japan

${ }^{6}$ Chiba Cardiovascular Center, Chiba, Japan

${ }^{7}$ Department of Neurosurgery, Kyorin University, Tokyo, Japan

${ }^{8}$ Showa University Hospital, Tokyo, Japan

${ }^{9}$ Department of Neurosurgery, Osaka Medical College, Osaka, Japan

${ }^{10}$ Kokura Memorial Hospital, Fukuoka, Japan

${ }^{11}$ Department of Cerebrovascular Medicine and Neurology, National Cerebral and Cardiovascular Center, Osaka, Japan

${ }^{12}$ Department of Preventive Medicine and Community Health, School of

Medicine, University of Occupational and Environmental Health, Fukuoka, Japan

${ }^{13}$ Akita Prefectural Hospital Organization Research Institute for Brain and Blood Vessels, Akita, Japan

${ }^{14}$ Department of Neurosurgery, National Cerebral and Cardiovascular Center, Suita, Japan

${ }^{15}$ Department of Public Health/Health Policy, Graduate School of Medicine, University of Tokyo, Tokyo, Japan

${ }^{16}$ Department of Neurosurgery, Graduate School of Medical Sciences, Kyushu University, Fukuoka, Japan
Acknowledgements The authors thank Dr Manabu Hasegawa for his cooperation on and designing of this study. They also thank Ms Arisa Ishitoko for secretarial assistance.

Contributors DO, AH and $\mathrm{KI}$ conceived and designed the experiments, analysed the data and wrote the manuscript. KN, AK, JN, KO, JO, YS, TA, SM, AS, HK, FN, SK, AN, RK and TS collected the data and advised on interpreting the results.

Funding This work was supported by Grants-in-Aid from the Ministry of Health, Labour and Welfare of Japan (principal investigator: KI).

Disclaimer The funders had no role in study design, data collection and analysis, decision to publish, or preparation of the manuscript.

Competing interests All authors had financial support from the Ministry of Health, Labour and Welfare of Japan for the submitted work. KI received research grants from Otsuka Pharmaceutical Co, Ltd, Nihon Medi-Physics Co, Ltd, and AstraZeneca KK. There are no other relationships or activities that could appear to have influenced the submitted work.

Ethics approval This study was approved by the ethics committee at Kyushu University Graduate School of Medical Sciences.

Provenance and peer review Not commissioned; externally peer reviewed.

Data sharing statement No additional data are available.

Open Access This is an Open Access article distributed in accordance with the Creative Commons Attribution Non Commercial (CC BY-NC 4.0) license, which permits others to distribute, remix, adapt, build upon this work noncommercially, and license their derivative works on different terms, provided the original work is properly cited and the use is non-commercial. See: http:// creativecommons.org/licenses/by-nc/4.0/

\section{REFERENCES}

1. Kuroda S, Houkin K. Moyamoya disease: current concepts and future perspectives. Lancet Neurol 2008;7:1056-66.

2. Scott RM, Smith ER. Moyamoya disease and moyamoya syndrome. N Engl J Med 2009;360:1226-37.

3. Research Committee on the Pathology and Treatment of Spontaneous Occlusion of the Circle of Willis; Health Labour Sciences Research Grant for Research on Measures for Intractable Diseases. Guidelines for diagnosis and treatment of moyamoya disease (spontaneous occlusion of the circle of Willis). Neurol Med Chir (Tokyo) 2012;52:245-66.

4. Suzuki J, Kodama N. Moyamoya disease-a review. Stroke 1983;14:104-9.

5. Caldarelli M, Di Rocco C, Gaglini P. Surgical treatment of moyamoya disease in pediatric age. J Neurosurg Sci 2001;45:83-91.

6. Wakai K, Tamakoshi A, Ikezaki K, et al. Epidemiological features of moyamoya disease in Japan: findings from a nationwide survey. Clin Neurol Neurosurg 1997;99(Suppl 2):S1-5.

7. Baba T, Houkin K, Kuroda S. Novel epidemiological features of moyamoya disease. J Neurol Neurosurg Psychiatry 2008;79:900-4.

8. Yonekawa Y, Ogata N, Kaku Y, et al. Moyamoya disease in Europe, past and present status. Clin Neurol Neurosurg 1997;99(Suppl 2): S58-60.

9. Hallemeier CL, Rich KM, Grubb RL Jr, et al. Clinical features and outcome in North American adults with moyamoya phenomenon. Stroke 2006;37:1490-6.

10. Uchino K, Johnston SC, Becker KJ, et al. Moyamoya disease in Washington State and California. Neurology 2005; 65:956-8.

11. Kraemer M, Berlit $P$, Diesner $F$, et al. What is the expert's option on antiplatelet therapy in moyamoya disease? Results of a worldwide Survey. Eur J Neurol 2012;19:163-7.

12. Shinohara Y. Japanese Guidelines for Management of Stroke, 2004 -present significance and future issues. Nihon Rinsho 2006;64 (Suppl 7):19-24.

13. Jo KI, Yeon JY, Hong SC, et al. Clinical course of asymptomatic adult moyamoya disease. Cerebrovasc Dis 2014;37:94-101.

14. DeVeber G. In pursuit of evidence-based treatments for paediatric stroke: the UK and Chest guidelines. Lancet Neurol 2005;4:432-6.

15. lihara K, Nishimura K, Kada A, et al. The impact of comprehensive stroke care capacity on the hospital volume of stroke interventions: 
a nationwide study in Japan: J-ASPECT study. J Stroke Cerebrovasc Dis 2014;23:1001-18.

16. Nishimura K, Nakamura F, Takegami M, et al. Cross-sectional survey of workload and burnout among Japanese physicians working in stroke care: the nationwide survey of acute stroke care capacity for proper designation of Comprehensive Stroke Center in Japan (J-ASPECT) study. Circ Cardiovasc Qual Outcomes 2014;7:414-22.

17. lihara K, Nishimura K, Kada A, et al. Effects of comprehensive stroke care capabilities on in-hospital mortality of patients with ischemic and hemorrhagic stroke: J-ASPECT study. PLOS ONE 2014;9:e96819.

18. Fukui M. Guidelines for the diagnosis and treatment of spontaneous occlusion of the circle of Willis ('moyamoya' disease). Research Committee on Spontaneous Occlusion of the Circle of Willis (Moyamoya Disease) of the Ministry of Health and Welfare, Japan. Clin Neurol Neurosurg 1997;99(Suppl 2):S238-40.

19. Smith ER, Scott RM. Spontaneous occlusion of the circle of Willis in children: pediatric moyamoya summary with proposed evidence-based practice guidelines. A review. J Neurosurg Pediatr 2012;9:353-60.

20. Kleinloog R, Regli L, Rinkel GJ, et al. Regional differences in incidence and patient characteristics of moyamoya disease: a systematic review. J Neurol Neurosurg Psychiatry 2012;83:531-6.

21. Kim JE, Jeon JS. An update on the diagnosis and treatment of adult moyamoya disease taking into consideration controversial issues. Neurol Res 2014;36:407-16.

22. Fujimura M, Tominaga T. Diagnosis of moyamoya disease: international standard and regional differences. Neurol Med Chir (Tokyo) 2015;55:189-93

23. Hoh BL, Chi YY, Waters MF, et al. Effect of weekend compared with weekday stroke admission on thrombolytic use, in-hospital mortality, discharge disposition, hospital charges, and length of stay in the Nationwide Inpatient Sample Database, 2002 to 2007. Stroke 2010;41:2323-8.

24. Bray BD, Ayis S, Campbell J, et al. Associations between stroke mortality and weekend working by stroke specialist physicians and registered nurses: prospective multicentre cohort study. PLoS Med 2014;11:e1001705.

25. Shigematsu K, Nakano $\mathrm{H}$, Watanabe $\mathrm{Y}$. The eye response test alone is sufficient to predict stroke outcome-reintroduction of Japan Coma Scale: a cohort study. BMJ Open 2013;3:pii: e002736.

26. Miyamoto S, Yoshimoto T, Hashimoto N, et al. Effects of extracranial-intracranial bypass for patients with hemorrhagic moyamoya disease: results of the Japan Adult Moyamoya Trial. Stroke 2014;45:1415-21.

27. Quan $\mathrm{H}, \mathrm{Li}$ B, Couris $\mathrm{CM}$, et al. Updating and validating the Charlson Comorbidity Index and score for risk adjustment in hospital discharge abstracts using data from 6 countries. Am J Epidemiol 2011:173:676-82.

28. Alberts MJ, Latchaw RE, Selman WR, et al. Recommendations for Comprehensive Stroke Centers: a consensus statement from the Brain Attack Coalition. Stroke 2005;36:1597-616.

29. Emberson J, Lees KR, Lyden P, et al, Stroke Thrombolysis Trialists' Collaborative Group. Effect of treatment delay, age, and stroke severity on the effects of intravenous thrombolysis with alteplase for acute ischaemic stroke: a meta-analysis of individual patient data from randomised trials. Lancet 2014;384:1929-35.

30. Wyss R, Ellis AR, Brookhart MA, et al. The role of prediction modeling in propensity score estimation: an evaluation of logistic regression, bCART, and the covariate-balancing propensity score. Am J Epidemiol 2014;180:645-55.

31. Joffe MM, Rosenbaum PR. Invited commentary: propensity scores. Am J Epidemiol 1999;150:327-33.

32. D'Agostino RB Jr. Tutorial in biostatistics: propensity score methods for bias reduction in the comparison of a treatment to a non-randomized control group. Stat Med 1998;17:2265-81.

33. Normand ST, Landrum MB, Guadagnoli E, et al. Validating recommendations for coronary angiography following acute myocardial infarction in the elderly: a matched analysis using propensity scores. J Clin Epidemiol 2001;54:387-98.

34. Cecchi AC, Guo D, Ren Z, et al. rnf213 rare variants in an ethnically diverse population with moyamoya disease. Stroke 2014:45:3200-7.

35. Sandercock PA, Counsell C, Tseng MC, et al. Oral antiplatelet therapy for acute ischaemic stroke. Cochrane Database Syst Rev 2014;3:CD000029.

36. Chen J, Duan L, Xu WH, et al. Microembolic signals predict cerebral ischaemic events in patients with moyamoya disease. Eur J Neurol 2014;21:785-90.

37. Iguchi $\mathrm{Y}$, Kimura K, Tateishi $\mathrm{Y}$, et al. Microembolic signals are associated with progression of arterial lesion in Moyamoya disease: a case report. J Neurol Sci 2007;260:253-5.

38. Kraemer M, Heienbrok W, Berlit P. Moyamoya disease in Europeans. Stroke 2008;39:3193-200.

39. Horn P, Lanczik O, Vajkoczy $\mathrm{P}$, et al. Hemodynamic reserve and high-intensity transient signals in moyamoya disease. Cerebrovasc Dis 2005;19:141-6.

40. Soman T, Rafay MF, Hune S, et al. The risks and safety of clopidogrel in pediatric arterial ischemic stroke. Stroke 2006;37:1120-2.

41. Zaidat OO, Fitzsimmons BF, Woodward BK, et al. Effect of a balloon-expandable intracranial stent vs medical therapy on risk of stroke in patients with symptomatic intracranial stenosis: the VISSIT randomized clinical trial. JAMA 2015;313:1240-8.

42. Holmstedt CA, Turan TN, Chimowitz Ml. Atherosclerotic intracranial arterial stenosis: risk factors, diagnosis, and treatment. Lancet Neurol 2013;12:1106-14.

43. Chimowitz MI, Lynn MJ, Derdeyn CP, et al. Stenting versus aggressive medical therapy for intracranial arterial stenosis. $N$ Engl $J$ Med 2011;365:993-1003.

44. Derdeyn CP, Chimowitz MI, Lynn MJ, et al. Aggressive medical treatment with or without stenting in high-risk patients with intracranial artery stenosis (SAMMPRIS): the final results of a randomised trial. Lancet 2014;383:333-41.

45. Johnston SC, Gress DR, Browner WS, et al. Short-term prognosis after emergency department diagnosis of TIA. JAMA 2000;284:2901-6.

46. Lovett JK, Coull AJ, Rothwell PM. Early risk of recurrence by subtype of ischemic stroke in population-based incidence studies. Neurology 2004:62:569-73.

47. Diener HC, Bogousslavsky J, Brass LM, et al. Aspirin and clopidogrel compared with clopidogrel alone after recent ischaemic stroke or transient ischaemic attack in high-risk patients (MATCH): randomised, double-blind, placebo-controlled trial. Lancet 2004;364:331-7.

48. Han $\mathrm{DH}, \mathrm{Nam} \mathrm{DH}$, Oh CW. Moyamoya disease in adults: characteristics of clinical presentation and outcome after encephalo-duro-arterio-synangiosis. Clin Neurol Neurosurg 1997;99 (Suppl 2):S151-5

49. Choi JU, Kim DS, Kim EY, et al. Natural history of moyamoya disease: comparison of activity of daily living in surgery and non surgery groups. Clin Neurol Neurosurg 1997;99(Suppl 2):S11-18.

50. Tsai CF, Thomas B, Sudlow CL. Epidemiology of stroke and its subtypes in Chinese vs White populations: a systematic review. Neurology 2013;81:264-72.

51. Kim BJ, Kim JS. Ischemic stroke subtype classification: an Asian viewpoint. J Stroke 2014;16:8-17.

52. Bang OY, Ryoo S, Kim SJ, et al. Adult moyamoya disease: a burden of intracranial stenosis in East Asians? PLOS ONE 2015;10: e0130663.

53. Koizumi A, Kobayashi H, Liu W, et al. P.R4810K, a polymorphism of RNF213, the susceptibility gene for moyamoya disease, is associated with blood pressure. Environ Health Prev Med 2013;18:121-9.

54. Yang J, Hong JC, Oh CW, et al. Clinicoepidemiological features of asymptomatic moyamoya disease in adult patients. $J$ Cerebrovasc Endovasc Neurosurg 2014;16:241-6. 